\title{
Paranasal Sinus Mucocele
}

\author{
Gregory G. Capra $\cdot$ Peter N. Carbone • \\ David P. Mullin
}

Received: 13 April 2012/Accepted: 28 April 2012/Published online: 24 May 2012

(C) Springer Science+Business Media, LLC (Outside the USA) 2012

\begin{abstract}
Paranasal sinus mucoceles are epithelium-lined cystic masses usually resulting from obstruction of sinus ostia. They most frequently occur in the frontal and ethmoid sinuses. While ophthalmologic symptoms are most common, patients also report rhinological or neurological complaints. The close proximity of paranasal sinus mucoceles to the orbit and skull base predisposes the patient to significant morbidity. Computed tomography displays a non-enhancing homogenous mass with expansion of bony walls. Magnetic resonance imaging reveals variable intensity of T1-weighted images and a hyperintense mass on T2-weighted images. Histopathologically mucoceles have features of respiratory mucosa with areas of reactive bone formation, hemorrhage, fibrosis, and granulation tissue. Surgical excision is the standard treatment with trends towards endoscopic techniques.
\end{abstract}

Keywords Paranasal sinus mucocele $\cdot$ Magnetic resonance $\cdot$ Computed tomography $\cdot$ Blindness . Ophthalmologic · Optic neuropathy · Cranial neuropathy · Osteolysis $\cdot$ Inflammatory infiltrate

The opinions and assertions expressed herein are those of the author and are not to be construed as official or representing the views of the Department of the Navy or the Department of Defense.

G. G. Capra $(\bowtie) \cdot$ D. P. Mullin

Department of Otolaryngology, Naval Medical Center San

Diego, 34800 Bob Wilson Drive, San Diego, CA 92134-5000,

USA

e-mail: gregory.capra@med.navy.mil

P. N. Carbone

Department of Anatomic Pathology, Naval Medical Center San

Diego, San Diego, CA, USA

\section{History}

A 54-year-old female with a history of cataracts was referred by an ophthalmologist to the Otolaryngology Clinic for acute worsening of visual acuity and diplopia in the right eye. Symptoms began 5 months prior and were described as a 'white curtain' in her vision. Her ophthalmologic exam showed proptosis and chemosis of the eye and her visual acuity ipsilaterally was 20/100 (baseline 20/25). The optic disc was pink and healthy on exam. Cranial nerves III, IV, V, and VI were intact, with only minimal impairment of extraocular muscle movement.

\section{Radiographic Features}

A non-contrast computerized tomographic (CT) scan of the head and orbits showed a $4.4 \mathrm{~cm} \times 4.2 \mathrm{~cm} \times 3.3 \mathrm{~cm}$ well-circumscribed expansile mass in the right ethmoid sinus. The medial orbital wall was expanded with evidence of mass effect upon the medial rectus muscle and optic nerve (Fig. 1). Nuclear magnetic resonance images (MRI) of the head with and without contrast demonstrated a hyperintense mass on T2-weighted images (WI) and a hypointensity on T1WI. The MR fluid attenuation inversion recovery (FLAIR) sequence suggested proteinaceous fluid (Fig. 2).

\section{Treatment}

The mass was emergently decompressed through an endoscopic approach in the operating room. Resistance to retropulsion of the right eye resolved immediately and the patient's visual acuity returned to baseline within 24-h of surgery. 


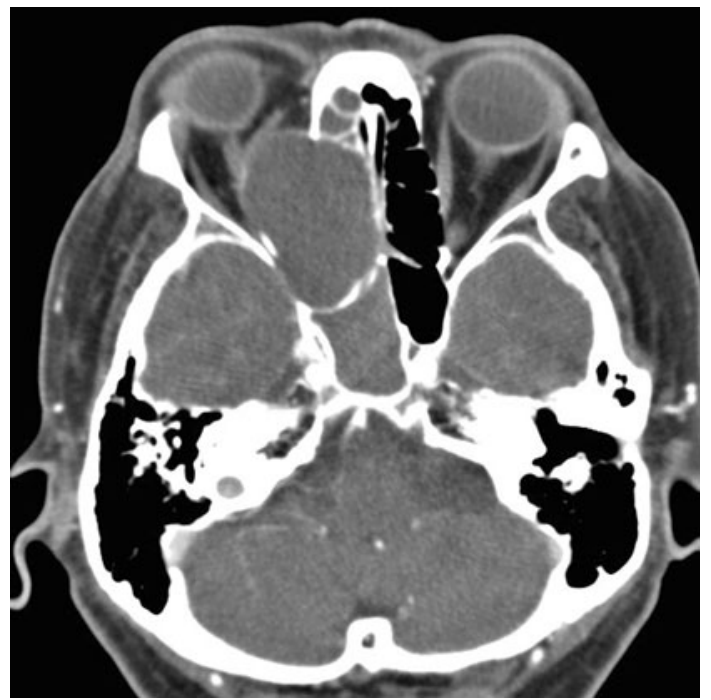

Fig. 1 Axial images of CT scan of orbits with contrast demonstrating a $4.4 \times 4.2 \times 3.3 \mathrm{~cm}$ mass consistent with a right ethmoid mucocele. The medial orbital wall has expanded and mass effect on the medial rectus and optic nerve is noted. Right-sided proptosis is also present

\section{Diagnosis}

Histological examination of a hematoxylin and eosin stained slide demonstrated benign respiratory mucosa with mild chronic inflammatory infiltrate and areas of reactive bone (Figs. 3, 4).

\section{Discussion}

Paranasal sinus mucoceles are epithelium-lined cystic masses, are mucus filled, and result from obstruction of sinus ostia. Mucus accumulation causes enlargement of the mass with associated sinus bony wall expansion that is considered to be sine qua non for this entity. Prostaglandins and collagenases aid in osteolysis and further enhance the expansile nature of the cysts [1-3]. Except in the case of pyeloceles, they are generally benign and slow growing with the potential, through mass effect, to cause local morbidity upon neighboring structures $[1,4,5]$.

Paranasal sinus mucoceles most commonly occur in the third or fourth decades of life with a slight male predilection $[3,5]$. The frontal sinus is most commonly affected followed by the ethmoid sinuses, with reports suggesting $70-90 \%$ of mucoceles occur in these locations. Ten percent of mucoceles occur in the maxillary sinus and the sphenoid sinus is involved rarely [3-6]. The general mechanisms of cyst formation are inflammation, trauma, or tumor distortion of sinus outflow tracts. In addition to inflammatory obstruction of ostia, primary causes also include cystic dilation of mucosal glands or polyp degeneration. Secondary causes most commonly result from previous sinus surgery or facial trauma. Other predisposing factors include cranial dysplasias, chronic sinusitis, and sinonasal manifestations of systemic disease [3, 4, 7]. In cystic fibrosis, $16 \%$ of patients with chronic rhinosinusits also have mucoceles [3].

Symptoms vary depending on the location of the mucocele and may be classified as rhinological, neurologic, or most frequently ophthalmologic [5, 7]. In general, fronto-ethmoidal involvement causes mass effect upon the orbit leading to proptosis, hypophthalmos, diplopia, and periorbital swelling. Conversely, visual compromise is more common with posterior ethmoid and sphenoid mucoceles because the thin walled lamina papyracea may be displaced into the optic canal by an expanding mucocele. Optic neuropathy may ensue from direct mechanical compression, ischemia, or optic neuritis secondary to inflammation. Other structures that may be involved
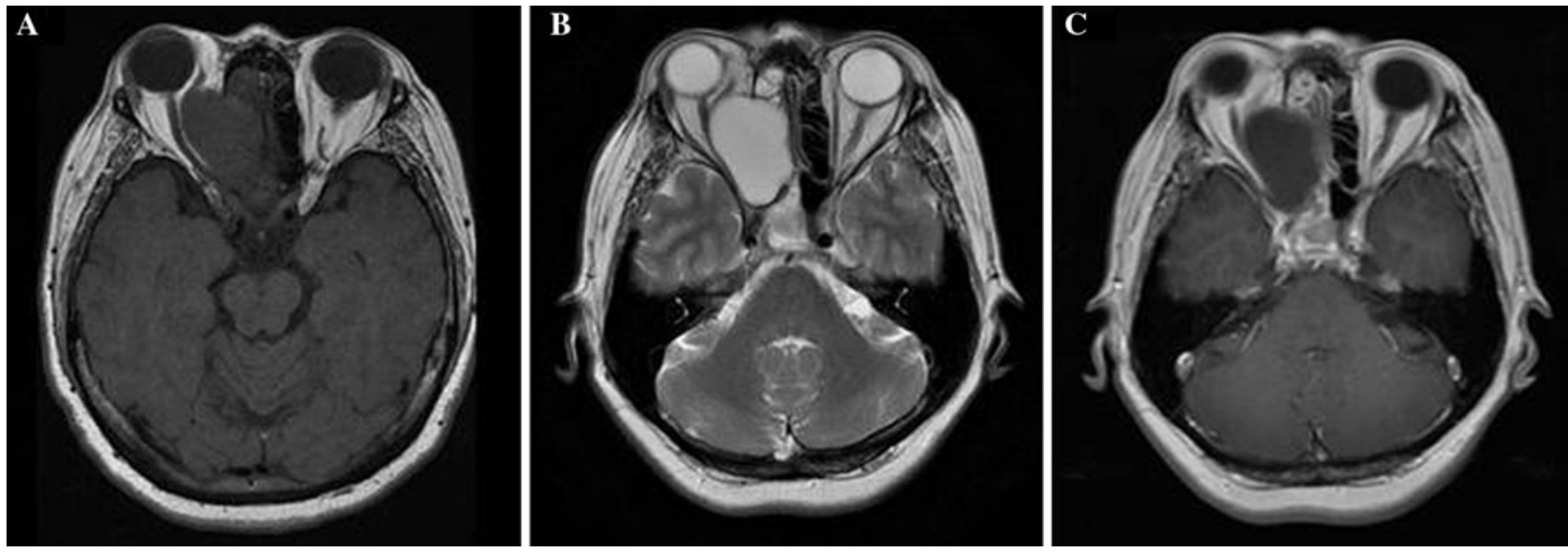

Fig. 2 Axial MR imaging of the brain with T1WI (a), T2WI (b), and contrast-enhanced T1WI (c) demonstrating an ethmoid mucocele causing mass effect on optic nerve and medial rectus 


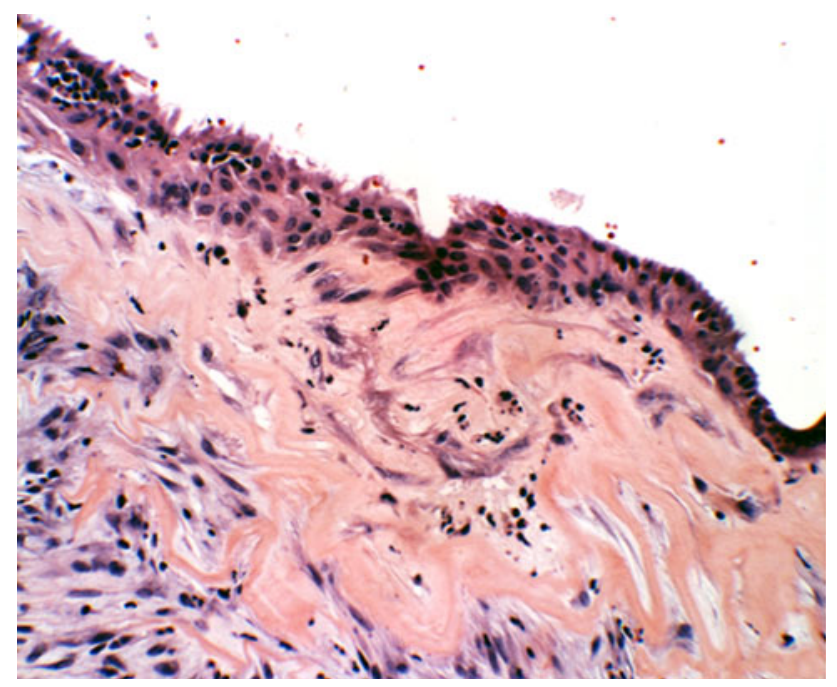

Fig. 3 Hematoxylin and eosin stain of ethmoid mucocele lined with ciliated pseudostratified respiratory-type epithelium with acute and chronic inflammatory infiltrates and fibrosis

include the third, fourth, fifth, and sixth cranial nerves and, rarely, the pituitary. Sphenoidal mucoceles are also more commonly associated with headaches $[1,7,8]$.

Radiographically, CT scans provide basic anatomical detail of the mucocele, delineate its interaction with neighboring bony structures, and aid in surgical planning. CT scan findings show an expansile, homogenous sinus mass that is not rim-enhancing, unless associated with an acute mucopylocele. Bony destruction is not common but expansion and remodeling of bone is seen in association with the mucocele $[2,4]$. MRI is superior in identifying the relationship of the mucocele to neighboring soft tissue and in distinguishing from other soft tissue neoplasms. Signal intensity of T1WI and T2WI is dependent upon the viscosity and fluid content of the cyst [5]. On T2WI, mucoceles are hyperintense owing to their high water content. With time, the intensity may diminish due to chronic inspissations. In contrast, T1WIs have low signal intensity initially but, with water absorption and increased protein concentration over time, a more viscous mucocele changes from an isointense to hyperintense structure [4].

Histologically, mucoceles within the paranasal sinus have features of respiratory mucosa with cyst walls demonstrating single-layered, pseudostratified, ciliated, columnar epithelium [2, 3, 8]. Although metaplastic changes are rare, chronic cases will show evidence of squamous metaplasia. Reactive bone formation is also possible in areas adjacent to cyst epithelium [2]. Mucoceles show elevated expression of IL-12, which secondarily leads to increased expression of IL-2 and interferon gamma. Subsequently, an increased activation of $\mathrm{T}_{\mathrm{H}} 2$ lymphocytes hastens the presence of a chronic inflammatory infiltrate [3]. Additional findings include cholesterol

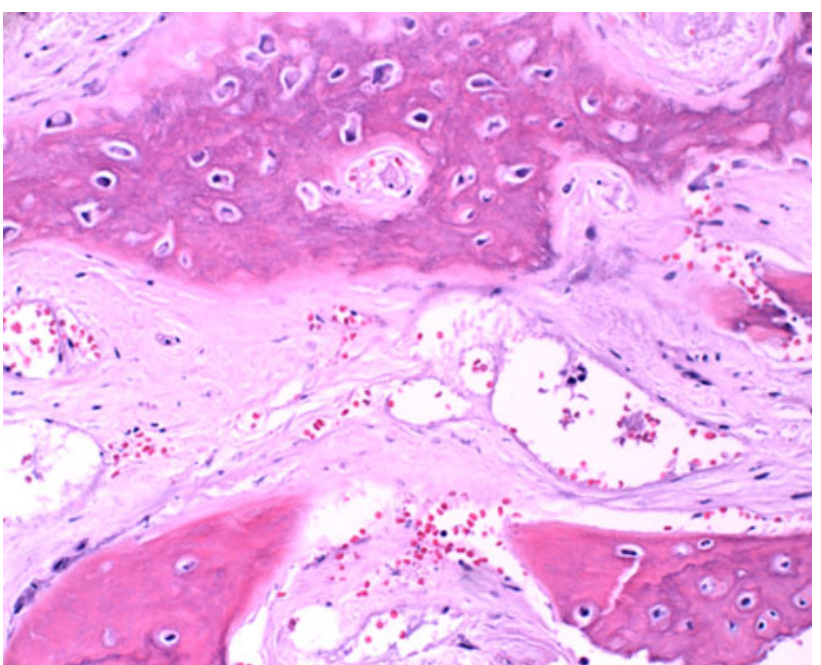

Fig. 4 Hematoxylin and eosin stain demonstrating osseous erosion associated with the ethmoid mucocele

granuloma, hemorrhage, fibrosis, and granulation tissue. Central nervous tissue may also be identified in those cases of intracranial herniation [2].

Surgical excision is the treatment of choice and early intervention is indicated to prevent visual compromise or in cases of pyelocele [1, 7]. Historically, mucoceles have been removed through external approaches, such as a LynchHowarth incision, Caldwell-Luc approach, or an osteoplastic flap. Recent trends have moved toward endoscopic management, when appropriate, and this has shown improved morbidity and recurrence rates [5, 7]. Overall recurrence rates vary among sources, but it is considered to be less than $10 \%$ of reported cases [3,5]. Optic neuropathy is the greatest concern and visual outcomes are generally good. Poorer outcomes are related to posterior ethmoid and sphenoidal mucoceles or delayed treatment of pyeloceles $[1,7]$.

Acknowledgments The authors would like to thank Dr. Brenda Nelson for her support and advice with writing this paper.

\section{References}

1. Loo JL, Looi AL, Seah LL. Visual outcomes in patient with paranasal mucoceles. Ophthal Plast Reconstr Surg. 2009;25:126-9.

2. Thompson LDR, Wenig BM. Mucocele of paranasal sinus. In: Diagnostic pathology: head and neck. Salt Lake City: Amirsys, 2011, p 45.

3. Obeso S, Llorente JL, Rodrigo JP, Sanchez R, Mancebo G, Suarez C. Paranasal sinuses mucoceles. Our experience in 72 patients. Acta Otorrinolaringol Esp. 2009;60(5):332-9.

4. Kao HW, Lo CP, Hsu YC, Chiu YC, Hsiao CH, Chen CY. Sphenoid sinus mucocele presenting with optic canal syndrome. J Med Sci. 2006;26(2):061-4.

5. Lee TJ, Li SP, Fu CH, Huang CC, Chang PH, Chen YW, Chen CW. Extensive paranasal sinus mucoceles: a 15-year review of 82 cases. Am J Otolaryngol Head Neck Med Surg. 2009;30:234-8. 
6. Yue CP, Mann KS, Chan FL. Optic canal syndrome due to posterior ethmoid sinus mucocele. J Neurosurg. 1986;65:871-3.

7. Kim YS, Kim K, Lee JG, Yoon JH, Kim CH. Paranasal sinus mucoceles with ophthalmologic manifestations: a 17-year review of 96 cases. Am J Rhinol Allergy. 2011;25:272-5.
8. Fukuda H, Fukumitsu R, Andoh M, Suzuki T, Yamana N, Kataoka H, Iwasaki K. Small onodi cell mucocele causing chronic optic neuropathy: case report. Neurol Med Chir (Tokyo). 2010;50: 953-5. 